\title{
Contribution of the 24 hour electrocardiogram to the prediction of sudden coronary death
}

\author{
A Algra, J G P Tijssen, J R T C Roelandt, J Pool, J Lubsen
}

\begin{abstract}
Background-Sudden coronary death is a major public health issue. The identification of patients at high risk should therefore be as efficient as possible. This study compares simple and more elaborate risk stratification procedures. Methods-Risk functions for the prediction of sudden death were determined in a population of 6693 consecutive patients who had 24 hour electrocardiography for various indications. The functions were based on the clinical and electrocardiographical data on 245 patients who died suddenly during 2 year follow up and 467 patients randomly drawn from the total study population.
\end{abstract}

Results-The model based on history (age, sex, myocardial infarction, congestive heart failure, palpitation, syncope, use of diuretics, and use of nitrates), 12 lead electrocardiography (major intraventricular conduction defect, $T$ wave abnormality, and ST depression $\geqslant 0.05$ $\mathbf{m V}$ ), and standard rhythm analysis of 24 hour electrocardiography (ventricular tachycardia, frequent premature atrial complexes, sinus tachycardia $(>150$ min $^{-1}$ ), and atrial fibrillation) was almost as efficient in the prediction of sudden death as extended models that also contained information from exercise testing, echocardiography, ventriculography, and computer-aided re-analysis of 24 hour electrocardiography (QT and RR interval variability).

Conclusions-These results indicate that additional information from advanced function tests does little to increase the efficiency of prediction of sudden coronary death over that of tests based on history, the standard 12 lead electrocardiogram, and 24 hour electrocardiography.

Thoraxcenter,

Department of

Cardiology, Erasmus

University Rotterdam

and University

Hospital Rotterdam-

Dijkzigt, Rotterdam,

The Netherlands

A Algra

J G P Tijssen

J R T C Roelandt

J Pool

J Lubsen

Correspondence to: Dr A Algra, Clinical Epidemiology Unit, Room X.00.123, University Hospital Utrecht, PO Box 85500,3508 GA Utrecht, The Netherlands.

Accepted for publication 31 March 1993
(Br Heart $\mathcal{F} 1993 ; 70: 421-427)$

Sudden coronary death is a major public health issue. There are more than 300000 such deaths every year in the United States. ${ }^{1}$ One of the main problems in the prevention of sudden coronary death is the identification of patients at high risk. Many single risk factors for sudden coronary death have been identified, ${ }^{2}$ however, little is known about the increase in the efficiency of the prediction of sudden death when information from more advanced tests is added to that derived from simple clinical characteristics.
In this paper we describe risk functions for the prediction of sudden death in patients who had 24 hour electrocardiography for various clinical conditions. We have evaluated their efficiency in identifying those patients most likely to die suddenly within two years of 24 hour electrocardiography. Risk functions were developed in a stepwise manner in the order in which clinical information becomes available. The first risk function was based only on the disease history. Subsequent risk functions included standard 12 lead electrocardiography, blood tests, data from standard rhythm analysis of 24 hour electrocardiograms, an exercise test, echocardiography, computer-aided analysis of 24 hour electrocardiograms, and contrast ventriculography.

\section{Patients and methods}

STUDY COHORT

We studied all 6693 consecutive patients who had 24 hour electrocardiography in one of the four participating hospitals between 1 August 1980 and 31 December 1984. Indications for 24 hour electrocardiography were the evaluation of symptoms potentially related to cardiac arrhythmias (palpitation, dizziness, syncope, angina) (65\%), of the effect of antiarrhythmic therapy $(8 \%)$, of risk after myocardial infarction (10\%), or a search for a cardiac cause of transient ischaemic attacks or strokes $(7 \%)$.

\section{FOLLOW UP}

Patients were followed for mortality in the two years after 24 hour ambulatory electrocardiography. Follow up was complete in $99.5 \%$ of the patients: 716 patients had died $(10.7 \%)$. The cause and circumstances of death were determined from the records of general practitioners and hospitals. Patients were considered to have died suddenly if death was observed and had occurred within one hour after new or more serious symptoms and if it was likely that the cause was cardiovascular. Also, patients who unexpectedly died during sleep or died while unobserved were considered to have died suddenly if circumstantial evidence pointed to sudden death from cardiovascular causes. All cases of sudden death were independently verified by two senior cardiologists. A total of 245 cases of sudden death was identified.

COLLECTION OF BASELINE DATA

Baseline characteristics were retrospectively collected for all patients who died suddenly and a random sample of 467 patients from 
the complete study cohort (including 21 cases of sudden death). A sample twice that of the sudden death group was chosen because of efficiency considerations. ${ }^{3}$ Information was collected from the medical records on the following patient characteristics at the time of 24 hour electrocardiography: known cardiovascular risk indicators, cardiovascular history, cardiovascular function tests if available, routine laboratory studies, current drug use, data on standard 12 lead electrocardiograms, data on standard rhythm analysis of the 24 hour electrocardiograms, and indices derived from computer-aided analysis of the original 24 hour electrocardiograms (Appendix A). Evidence of cardiac dysfunction was considered to be present if there had been a history of symptoms of pump failure or an ejection fraction $<40 \%$ at cineangiographic or radionuclide ventriculography. Details on analyses of the data from the standard 12 lead electrocardiograms and the computer-aided analysis have been reported elsewhere. ${ }^{3-5}$

\section{RISK FUNCTION DEVELOPMENT AND}

ASSESSMENT

The object of the analysis was to find the combination of baseline variables that most accurately predicted the occurrence of sudden death within two years of 24 hour electrocardiography. The most efficient and rational method of evaluating the risk of sudden death is a quantitative synthesis of the major risk factors for sudden death into a composite score. This was accomplished by the use of a logistic regression function (Appendix B).

Variables selected from univariate analysis (for details see Algra et $\mathrm{l}^{3}$ ) were sequentially entered into the model until no remaining candidate variable had a significance level of $0 \cdot 10$. For this purpose the SAS procedure LOGIST was used. ${ }^{6}$ For consecutive models we presented groups of variables for inclusion. For each model we calculated risk estimates for each patient to check whether the predicted risk accorded with the observed

Table 1 Sudden death in relation to presence or absence of selected baseline characteristics at the time of 24 hour electrocardiography

\begin{tabular}{|c|c|c|c|c|}
\hline \multirow[b]{2}{*}{ Characteristic } & \multicolumn{2}{|c|}{2 year sudden death rate } & \multirow[b]{2}{*}{$R R^{*}$} & \multirow[b]{2}{*}{$95 \% C I$} \\
\hline & $\begin{array}{l}\text { Present } \\
(\%(n))\end{array}$ & $\begin{array}{l}\text { Absent } \\
(\%(n))\end{array}$ & & \\
\hline \multicolumn{5}{|l|}{ Demographic characteristics: } \\
\hline Male & $4 \cdot 9(186 / 3812)$ & $2 \cdot 1(58 / 2795)$ & $2 \cdot 4$ & $1 \cdot 7$ to $3 \cdot 4$ \\
\hline Age $\geqslant 60$ & $5 \cdot 4(178 / 3325)$ & $2 \cdot 0(66 / 3282)$ & $2 \cdot 7$ & $2 \cdot 0$ to $4 \cdot 0$ \\
\hline \multicolumn{5}{|l|}{ History: } \\
\hline Angina & $6 \cdot 3(140 / 2207)$ & $2 \cdot 4(104 / 4400)$ & $2 \cdot 7$ & 2.0 to 3.9 \\
\hline Myocardial infarction & $7 \cdot 2(151 / 2092)$ & $2 \cdot 1(93 / 4515)$ & $3 \cdot 5$ & $2 \cdot 6$ to $5 \cdot 0$ \\
\hline Congestive heart failure & $9 \cdot 7(101 / 1046)$ & $2 \cdot 6(143 / 5561)$ & $3 \cdot 8$ & $2 \cdot 8$ to 5.9 \\
\hline Palpitation & $2.5(82 / 3311)$ & $4.9(162 / 3296)$ & 0.5 & 0.4 to 0.7 \\
\hline Syncope & $5 \cdot 0(58 / 1161)$ & $3.4(186 / 5446)$ & 1.5 & $1 \cdot 0$ to $2 \cdot 2$ \\
\hline Stroke & $5 \cdot 8(41 / 702)$ & $3.4(203 / 5905)$ & 1.7 & $1 \cdot 1$ to $2 \cdot 7$ \\
\hline Intermittent claudication & $6 \cdot 2(30 / 487)$ & $3.5(214 / 6120)$ & 1.8 & $1 \cdot 1$ to $3 \cdot 3$ \\
\hline Diabetes mellitus & $7 \cdot 5(40 / 530)$ & $3 \cdot 4(204 / 6077)$ & $2 \cdot 2$ & 1.4 to 3.8 \\
\hline \multicolumn{5}{|l|}{ Current use: } \\
\hline Cigarettes & $3 \cdot 6(83 / 2279)$ & $3 \cdot 7(161 / 4328)$ & $1 \cdot 0$ & 0.7 to 1.4 \\
\hline Alcohol $>6$ units/day & $7 \cdot 0(18 / 258)$ & $3.6(226 / 6349)$ & $2 \cdot 0$ & 1.0 to 3.7 \\
\hline Digitalis & $7 \cdot 8(121 / 1548)$ & $2 \cdot 4(123 / 5059)$ & $3 \cdot 2$ & $2 \cdot 4$ to $4 \cdot 7$ \\
\hline$\beta$ Blockers & $4 \cdot 3(70 / 1634)$ & $3 \cdot 5(174 / 4973)$ & $1 \cdot 2$ & 0.9 to 1.8 \\
\hline Nitrates & $6 \cdot 8(106 / 1548)$ & $2 \cdot 7(138 / 5059)$ & $2 \cdot 5$ & $2 \cdot 0$ to $4 \cdot 0$ \\
\hline Diuretics & $9 \cdot 3(143 / 1534)$ & $2 \cdot 0(101 / 5073)$ & $4 \cdot 7$ & $3 \cdot 4$ to $6 \cdot 8$ \\
\hline Antihypertensive drugs & $8 \cdot 0(47 / 588)$ & $3.3(197 / 6019)$ & $2 \cdot 4$ & $1 \cdot 6$ to $4 \cdot 1$ \\
\hline \multicolumn{5}{|l|}{ Standard 12 lead electrocardiography: } \\
\hline Major $Q$ waves $(1 \cdot 2+1 \cdot 3) \dagger$ & $8 \cdot 5(74 / 874)$ & $2 \cdot 5(110 / 4414)$ & $3 \cdot 4$ & $2 \cdot 4$ to $5 \cdot 5$ \\
\hline Major ST depression $(4 \cdot 1+4 \cdot 2)$ & $7 \cdot 5(78 / 1046)$ & $2 \cdot 7(122 / 4472)$ & $2 \cdot 7$ & $2 \cdot 1$ to $4 \cdot 5$ \\
\hline Negative or flat $T$ wave (5) & $5 \cdot 8(154 / 2666)$ & $2 \cdot 3(87 / 3741)$ & $2 \cdot 5$ & 1.9 to 3.7 \\
\hline Major ventricular conduction defect & $11 \cdot 1(43 / 387)$ & $3 \cdot 2(186 / 5747)$ & $3 \cdot 4$ & $2 \cdot 2$ to $6 \cdot 3$ \\
\hline CIIS $10-30 \S$ & $5 \cdot 2(112 / 2150)$ & $1 \cdot 3(42 / 3210)$ & $4 \cdot 0$ & $2 \cdot 8$ to $6 \cdot 4$ \\
\hline CIIS $\geqslant 30$ & $8.3(87 / 1046)$ & $1 \cdot 3(42 / 3210)$ & $6 \cdot 4$ & $4 \cdot 4$ to $11 \cdot 0$ \\
\hline QTc leads I, II, III (ms) & $5 \cdot 3(65 / 1233)$ & $2 \cdot 5(111 / 4357)$ & $2 \cdot 1$ & $1 \cdot 4$ to $3 \cdot 1$ \\
\hline \multicolumn{5}{|l|}{24 hour electrocardiography, standard rhythm analysis: } \\
\hline PVC \pm doublets & $3 \cdot 1(153 / 4859)$ & $1 \cdot 0(12 / 1161)$ & $3 \cdot 0$ & $1 \cdot 7$ to 5.9 \\
\hline Ventricular tachycardia $\mid$ & $11.9(80 / 674)$ & $1 \cdot 0(12 / 1161)$ & 11.5 & $6 \cdot 5$ to $27 \cdot 3$ \\
\hline Ventricular bigeminy & $6 \cdot 8(86 / 1261)$ & $2 \cdot 9(159 / 5432)$ & $2 \cdot 3$ & $1 \cdot 8$ to $3 \cdot 7$ \\
\hline Frequent PAC $(<30 \%)$ & $8 \cdot 5(40 / 473)$ & $3 \cdot 3(205 / 6220)$ & $2 \cdot 6$ & $1 \cdot 7$ to $4 \cdot 7$ \\
\hline Supraventricular tachycardia & $5 \cdot 3(92 / 1734)$ & $3 \cdot 1(153 / 4959)$ & $1 \cdot 7$ & $1 \cdot 3$ to $2 \cdot 5$ \\
\hline Atrial fibrillation & $7 \cdot 4(32 / 430)$ & $3.4(213 / 6263)$ & $2 \cdot 2$ & $1 \cdot 3$ to $3 \cdot 7$ \\
\hline Sinus arrhythmia & $2 \cdot 1(52 / 2508)$ & $4 \cdot 6(193 / 4185)$ & 0.4 & 0.3 to 0.6 \\
\hline Sinus bradycardia $\left(<50 \mathrm{~min}^{-1}\right)$ & $2 \cdot 4(42 / 1748)$ & $4 \cdot 1(203 / 4945)$ & $0 \cdot 6$ & 0.4 to 0.9 \\
\hline Sinus tachycardia $\left(>150 \mathrm{~min}^{-1}\right)$ & $0.6(4 / 645)$ & $4 \cdot 0(241 / 6048)$ & $0 \cdot 2$ & 0.1 to 0.4 \\
\hline \multicolumn{5}{|l|}{24 hour electrocardiography, computer-aided analysis: } \\
\hline Maximum heart rate $<100 \mathrm{~min}^{-1}$ & $5 \cdot 6(59 / 1056)$ & $2 \cdot 9(134 / 4594)$ & 1.9 & $1 \cdot 2$ to $3 \cdot 1$ \\
\hline Minimum heart rate $\geqslant 65 \mathrm{~min}^{-1}$ & $6 \cdot 2(69 / 1105)$ & $2 \cdot 7(124 / 4545)$ & $2 \cdot 3$ & 1.5 to 3.8 \\
\hline Short-term $R R$ variation $<25 \mathrm{~ms}$ & $7 \cdot 0(86 / 1228)$ & $2 \cdot 3(99 / 4250)$ & $3 \cdot 0$ & $2 \cdot 1$ to $5 \cdot 0$ \\
\hline Mean QTc $<400$ or $\geqslant 440 \mathrm{~ms}$ & $3 \cdot 1(65 / 2064)$ & $1 \cdot 3(36 / 2678)$ & $2 \cdot 3$ & 1.5 to 4.0 \\
\hline Maximum QTc $<440$ or $\geqslant 480 \mathrm{~ms}$ & $3 \cdot 4(64 / 1892)$ & $1 \cdot 3(40 / 3046)$ & $2 \cdot 6$ & 1.6 to 4.3 \\
\hline \multicolumn{5}{|l|}{ Cardiac function tests: } \\
\hline LV dilatation at echocardiography & $8 \cdot 7(109 / 1247)$ & $2 \cdot 5(135 / 5360)$ & $3 \cdot 5$ & $2 \cdot 6$ to $5 \cdot 3$ \\
\hline Maximum load exercise test $<70 \%$ & $10 \cdot 1(45 / 444)$ & $3 \cdot 2(199 / 6163)$ & $3 \cdot 1$ & 1.9 to $5 \cdot 1$ \\
\hline Ejection fraction $<40 \%$ & $22 \cdot 3(48 / 215)$ & $3 \cdot 1(196 / 6392)$ & $7 \cdot 3$ & $4 \cdot 0$ to $13 \cdot 8$ \\
\hline
\end{tabular}

$\mathrm{RR}$, relative risk; CI, confidence interval; AV, atrioventricular; CIIS; Cardiac Infarction Injury Score; PVC, premature ventricular complex; PAC, premature atrial complex.

${ }^{\star}$ Calculated from the ratio of sudden death rates in which the rate in the patients without the characteristic was taken as the ref* Calculated from the ratio of sudden death rates in which codes; fleft bundle branch block $(7 \cdot 1)$ or intraventricular block $(7 \cdot 4)$ or rence standard; tnumbers in past anterior fascicular block (7.8); \&see Rautaharju et al ${ }^{11}$, CIIS <10 was taken as reference; Ilabsence of PVC, or ventricular doublets, or tachycardia was taken as the reference standard. 
Table 2 Indicator variates retained in the logistic regression models for the prediction of sudden death on the basis of history, 12 lead electrocardiography, standard rhythm and computer-aided analysis of 24 hour electrocardiography, exercise test, echocardiography, and ventriculography

\begin{tabular}{|c|c|c|c|c|c|c|}
\hline \multirow[b]{2}{*}{ Indicator } & \multicolumn{2}{|c|}{$M 1(n=684)$} & \multicolumn{2}{|c|}{$M 2(n=667)$} & \multicolumn{2}{|c|}{$M 3(n=480)$} \\
\hline & Coefficient & $S E$ & Coefficient & $S E$ & Coefficient & $S E$ \\
\hline \multicolumn{7}{|l|}{ History: } \\
\hline Age $\geqslant 60$ years & 0.75 & $0 \cdot 21$ & $0 \cdot 35$ & $0 \cdot 22$ & 0.63 & $0 \cdot 27$ \\
\hline Male & 0.78 & 0.22 & 0.86 & 0.23 & 0.77 & 0.27 \\
\hline Myocardial infarction & 0.74 & 0.20 & 0.63 & $0 \cdot 22$ & 0.45 & 0.27 \\
\hline Congestive heart failure & $0 \cdot 70$ & 0.23 & 0.49 & 0.24 & - & - \\
\hline Palpitation & -0.47 & 0.20 & -0.59 & 0.21 & -0.62 & 0.25 \\
\hline Syncope & 0.65 & 0.23 & $0 \cdot 70$ & $0 \cdot 25$ & 0.82 & 0.30 \\
\hline Stroke & 0.48 & 0.27 & - & - & - & - \\
\hline Alcohol use $>6$ units/day & 0.69 & 0.41 & - & - & - & - \\
\hline \multicolumn{7}{|l|}{ Current drug use: } \\
\hline Diuretics & 1.03 & $0 \cdot 21$ & 0.78 & $0 \cdot 22$ & 0.76 & $0 \cdot 26$ \\
\hline Nitrates & 0.50 & 0.21 & 0.48 & $0 \cdot 22$ & - & - \\
\hline Antihypertensive drugs & 0.49 & 0.28 & - & - & - & - \\
\hline \multicolumn{7}{|l|}{12 lead electrocardiography: } \\
\hline Major IVCD* & & & 1.22 & 0.36 & 0.91 & 0.42 \\
\hline Flat or negative $T$ wave $f$ & & & 0.47 & $0 \cdot 27$ & 0.69 & 0.28 \\
\hline ST depression $\geqslant 0.05 \mathrm{~m} \mathrm{~V} \ddagger$ & & & 0.51 & $0 \cdot 26$ & - & - \\
\hline \multicolumn{7}{|c|}{24 hour electrocardiography, standard rhythm analysis: } \\
\hline Ventricular tachycardia & & & 0.87 & $0 \cdot 26$ & 0.96 & 0.32 \\
\hline Frequent PAC ( $>30 \%)$ & & & $1 \cdot 23$ & 0.32 & $1 \cdot 13$ & 0.38 \\
\hline Sinus tachycardia $\left(>150 \mathrm{~min}^{-1}\right)$ & & & -1.03 & 0.62 & - & - \\
\hline Atrial fibrillation & & & 0.61 & 0.34 & - & - \\
\hline \multicolumn{7}{|c|}{24 hour electrocardiography, computer-aided analysis: } \\
\hline Maximum heart rate $<100 \mathrm{~min}^{-1}$ & & & & & $0 \cdot 70$ & $0 \cdot 28$ \\
\hline Maximum QTc $<440$ or $\geqslant 480 \mathrm{~ms}$ & & & & & 0.52 & 0.25 \\
\hline \multicolumn{7}{|l|}{ Exercise test: } \\
\hline Max load $<70 \%$ norm or not available & & & & & $1 \cdot 12$ & $0 \cdot 28$ \\
\hline \multicolumn{7}{|l|}{ Echocardiography: } \\
\hline Dilatation of left ventricle (EDD $\geqslant 55 \mathrm{~mm}$ ) & & & & & $1 \cdot 14$ & $0 \cdot 28$ \\
\hline \multicolumn{7}{|l|}{ Ventriculography: } \\
\hline Ejection fraction $<40 \%$ & & & & & $1 \cdot 05$ & 0.46 \\
\hline Constant§ & $-5 \cdot 49$ & & $-5 \cdot 72$ & & $-7 \cdot 02$ & \\
\hline
\end{tabular}

M1, history only; M2, M1 + 12 lead electrocardiography + standard rhythm analysis of 24 hour electrocardiography; $M 3$ all data M1, history only; $M 2, M 1+12$ lead electrocardiography + standard rhythm analysis of 24 hour electrocardiography; M3 all data
available; $M$, model; IVCD, intraventricular conduction defect; - , variable did not meet significance level for entry; EDD, end diastolic diameter.

${ }^{\star}$ Left bundle branch block or intraventricular block or right bundle branch block with left anterior fascicular block (Minnesota codes $7.1,7.4$, and 7.8); † Minnesota code 5 ; $¥$ Minnesota code 4.1 or 4.2 ; $\$$ corrected to enable the calculation of absolute risks, see appendix B.

risk. For this purpose patients were grouped into five subgroups according to estimated risk: low, medium low, medium, medium high, and high risk. Each subgroup contained about the same number of cases. For each subgroup the mean of the individual risk estimates was compared with the observed risk. ${ }^{7}$ We used receiver-operator characteristic (ROC) curves to compare the information content of the models. ${ }^{8}$ The more a ROC curve is located in the upper left corner of the graph the higher the information content of the risk function is-that is, the higher both the sensitivity and specificity for the prediction of sudden death.

\section{Results}

\section{UNIVARIATE ANALYSIS}

Table 1 shows the occurrence of sudden death in relation to selected baseline characteristics at the time of 24 hour electrocardiography. Age $\geqslant 60$ years, male sex, history of angina, myocardial infarction, congestive heart failure, and a ventricular aneurysm clearly increased the risk for sudden death with relative risk estimates ranging from $2 \cdot 4$ to 4.5 . Patients complaining of palpitation had half the risk of sudden death as those without. Patients with a history of stroke, intermittent claudication, or diabetes mellitus had an approximately twofold risk for sudden death. Patients using digitalis, nitrates, diuretics, or antihypertensive drugs also had a clearly higher risk. Indices of poor left ventricular function as expressed by left ventricu- lar dilatation at echocardiography (end diastolic diameter $\geqslant 55 \mathrm{~mm}^{9}$ ), a low maximum work load at bicycle exercise testing, or an ejection fraction $<40 \%$ had clear risk implications.

Virtually all classification items of the Minnesota code ${ }^{10}$ except code 2, were related to sudden death. Patients with a normal Cardiac Infarction Injury Score (CIIS ${ }^{11}$ ) that is, $<10$ - had a low risk for sudden death $(1 \cdot 3 \%)$, while patients with scores between 10 and 30 had a fourfold and those with a score $>30$ had a sixfold risk. The lower part of the table shows the occurrence of sudden death in relation to the indices derived from standard rhythm analysis of the 24 hour electrocardiograms. Patients without premature ventricular complexes, ventricular doublets, or ventricular tachycardia were at low risk for sudden death $(1 \cdot 0 \%)$. Those with premature ventricular complexes or ventricular doublets had a three times higher risk and those with ventricular tachycardia had a more than tenfold increase in risk. Patients with frequent premature atrial complexes-that is, occurring during $30 \%-90 \%$ of the recording time-and those with atrial fibrillation had twice the risk for sudden death as those without these supraventricular arrhythmias. Patients with sinus arrhythmia, sinus bradycardia $\left(<50 \mathrm{~min}^{-1}\right)$, or sinus tachycardia (>150 $\mathrm{min}^{-1}$, detected in episodes in which heart rate gradually increased and decreased and $P$ waves could be detected) had lower risks. The relation of sudden death to selected RR and QTc interval indices derived 
Table 3 Risk of sudden death within two years of 24 hour electrocardiography predicted by logistic models 1-3 and observed risk

\begin{tabular}{|c|c|c|c|c|c|c|}
\hline \multirow[b]{2}{*}{ Risk score } & \multicolumn{2}{|c|}{$M 1(n=684)$} & \multicolumn{2}{|c|}{$M 2(n=667)$} & \multicolumn{2}{|c|}{$M 3(n=480)$} \\
\hline & Pred & Obs & Pred & Obs & Pred & Obs \\
\hline $\begin{array}{l}\text { Low risk } \\
\text { Medium low risk } \\
\text { Medium risk } \\
\text { Medium high risk } \\
\text { High risk }\end{array}$ & $\begin{array}{r}1 \cdot 1 \\
3 \cdot 8 \\
7 \cdot 6 \\
13 \cdot 7 \\
26 \cdot 0\end{array}$ & $\begin{array}{r}1 \cdot 2 \\
3 \cdot 4 \\
7 \cdot 3 \\
12 \cdot 5 \\
31 \cdot 1\end{array}$ & $\begin{array}{r}1 \cdot 0 \\
4 \cdot 7 \\
9 \cdot 2 \\
15 \cdot 6 \\
35 \cdot 4\end{array}$ & $\begin{array}{r}1 \cdot 2 \\
3 \cdot 6 \\
10 \cdot 5 \\
19 \cdot 7 \\
28 \cdot 5\end{array}$ & $\begin{array}{r}1 \cdot 2 \\
5 \cdot 2 \\
10 \cdot 6 \\
19 \cdot 1 \\
47 \cdot 9\end{array}$ & $\begin{array}{r}1 \cdot 0 \\
5 \cdot 1 \\
10 \cdot 9 \\
14 \cdot 0 \\
65 \cdot 1\end{array}$ \\
\hline
\end{tabular}

Pred, mean of predicted risks for the patients within a risk category; Obs, observed risk for the patients within a risk category. from computer-aided analysis of 24 hour electrocardiograms is shown at the bottom of table 1. A low maximum $\left(<100 \mathrm{~min}^{-1}\right)$ and a high minimum ( $\geqslant 65 \mathrm{~min}^{-1}$ ) heart rate as well as a lack of short-term heart rate variability ( $<25 \mathrm{~ms}$ ) increased the risk of sudden death, as did extremes of mean and maximum QTc duration.

\section{MULTIVARIATE RISK FUNCTIONS}

Table 2 summarises three logistic regression models. Model 1 was developed with the use of history only; in model 2 data from standard 12 lead electrocardiography and standard rhythm analysis of 24 hour electrocardiography were added. Common laboratory tests and the cardiothoracic ratio did not reach the significance level required for inclusion when they were added to model 2. Model 2 was extended with data from computer-aided analysis of 24 hour electrocardiography, exercise test, echocardiography, and contrast ventriculography (model 3).

Model 1 was based on 684 patients (21 sudden death patients were selected for the random sample; data for seven patients were incomplete). Model 2 was based on 667 patients because standard 12 lead electrocardiograms were not available in 17 patients. Model 3, including indices derived from the computer-aided analysis of 24 hour electrocardiograms, was based on 480 patients because not all 24 hour electrocardiograms had been subjected to the labour intensive computer-aided analysis (Appendix A). Table 3 shows the predicted and observed risks for sudden death for each of the models 1,2 and

Receiver-operator characteristic curves for prediction models 1-3.
3. The Figure shows receiver-operator characteristic (ROC) curves based on models 1-3.

\section{Discussion}

STEPWISE APPROACH

The risk functions were developed according to a stepwise approach that followed clinical decision making in practice. Disease history is always available to the cardiologist and therefore was used for the basic model 1 . Because our study population was defined by 24 hour electrocardiography we judged that data from the standard 12 lead electrocardiogram and standard rhythm analysis of 24 hour electrocardiography should be added next rather than data from bicycle exercise testing and echocardiography. Information from these latter tests and that from contrast ventriculography was entered in the final model (model 3).

PREDICTION MODEL BASED ON HISTORY ONLY In model 1 age $\geqslant 60$ years and male sex each independently doubled the risk for sudden death within two years after 24 hour electrocardiography. These data correspond well with those from the Framingham study which showed in univariate analysis that males had approximately a 2.5 higher risk for sudden death than females, irrespective of the presence of coronary heart disease. ${ }^{12}$ Risk in both sexes increased with age. ${ }^{12}$ These relations may be explained by a higher prevalence of atherosclerotic heart disease in men and older people. The known risk factors for cardiovascular disease cigarette smoking, hypertension, hypercholesterolaemia, diabetes mellitus, and family history did not enter the model. The presence of overt heart disease in a large part of the study population probably prevailed over these risk factors. A history of myocardial infarction, stroke, and nitrate use were all included in model 1 and may be interpreted as indicators of advanced atherosclerotic heart disease. Use of diuretics had the strongest independent relation with sudden death in this model (relative risk 2.8). The use of diuretics and the presence of a history of congestive heart failure were both retained in the model; this may be because of the use of diuretics as antihypertensive drugs but may also be attributed to the potassium depleting effects of the thiazide diuretics that can predispose patients to lethal arrhythmias. ${ }^{13}$ No conclusions on the relation between drug use and sudden death should be derived from our study because patients with a high risk for sudden death are more likely to have been given such treatment ("confounding by indication").

A history of palpitation was negatively related to sudden death, as reported by Velema. ${ }^{14} \mathrm{We}$ are not aware of any published pathophysiological explanation for this intriguing finding. A history of syncope must be taken seriously; patients with such a history have about twice the risk of sudden death, which is independent of the other variables taken into account. 


\section{PREDICTION MODEL BASED ON HISTORY AND} ECG DATA

Model 2 showed a strong independent relation between sudden death and left bundle branch block, right bundle branch block combined with a left anterior fascicular block, and intraventricular block (QRS duration $\geqslant 120$ ms) (relative risk 3.2). These findings accord with those from other studies. These conduction defects are regarded as a manifestation of advanced atherosclerotic heart disease. ${ }^{1516}$ Ventricular tachycardia showed a strong independent relation with sudden death. This relation has often been reported in patients after myocardial infarction ${ }^{17-22}$ and also in another study on patients who had 24 hour electrocardiography. ${ }^{14} 23$ Ventricular tachycardia indicates a heart at high risk because the myocardium (substrate) is impaired or modulating factors are present. ${ }^{24}{ }^{25}$ Frequent premature atrial complexes were an even stronger independent predictor of sudden death. To the best of our knowledge this finding has never been reported before. A direct relation between premature atrial complexes and the occurrence of sudden death is difficult to explain. Increased diastolic pressure as a result of ischaemic heart disease can cause supraventricular ectopic activity and atrial fibrillation, however. Our data do not support this hypothesis because frequent premature atrial complexes had predictive value independent of indices of left ventricular dysfunction. Patients with sinus tachycardia $(>150$ $\mathrm{min}^{-1}$ ) had a lower risk of sudden death than those without. This relation probably shows that patients in good physical condition without overt heart disease are able to do heavy exercise, and do.

\section{EXTENDED PREDICTION MODEL}

In the expanded model 3 both a low maximum exercise load and left ventricular dilatation at echocardiography were retained as well as a low ventricular ejection fraction. These three variables, all indicating impaired cardiac function, superseded the importance of a history of congestive heart failure. The fact that all three together entered the model may indicate that they all carry some independent prognostic information. Several variables that just failed to reach statistical significance were dropped from model 2: nitrates, ST depression, sinus tachycardia $\left(>150 \mathrm{~min}^{-1}\right)$, and atrial fibrillation. However, this was largely because model 3 was based on fewer patients than model $2(480 v 667$ patients).

Table 4 Variables of $Q T c$ and $R R$ duration and variability during 24 hour electrocardiography and their definition

\begin{tabular}{|c|c|}
\hline Variable & Definition \\
\hline $\begin{array}{l}\text { RR interval / heart rate: } \\
\text { Maximum heart rate } \\
\text { Minimum heart rate } \\
\text { Short-term variation } R R\end{array}$ & $\begin{array}{l}\text { Maximum of per minute heart rate means } \\
\text { Minimum of per minute heart rate means } \\
\text { Mean over } 24 \text { hours of per minute standard } \\
\text { deviations of RR intervals }\end{array}$ \\
\hline $\begin{array}{l}\text { QTc interval: } \\
\text { Mean QTc } \\
\text { Maximum QTc }\end{array}$ & $\begin{array}{l}\text { Mean over } 24 \text { hours of per minute QTc means } \\
\text { Maximum of per minute QTc means }\end{array}$ \\
\hline
\end{tabular}

INFORMATION CONTENT, RISK FUNCTIONS, AND ASSESSMENT OF FIT

First, risk functions were constructed based on routinely available clinical indices. In the extended model variables from more advanced (and more expensive) tests were added. We used the ROC curves (figure) to evaluate the increasing information content of the models. Model 2 clearly contained more information than model 1 , but the extended model (model 3 ) did not contain much more information than model 2 , which was based on history, standard 12 lead electrocardiogram, and standard rhythm analysis of 24 hour electrocardiograms. Thus relatively simple clinical indices predicted sudden death as reliably as models to which information from more advanced tests was added.

The models we developed showed in general a good correspondence between the predicted and observed risk for sudden death (table 3). However, this result may be partly attributable to the fact that the fit was assessed in the same patients as those in which the models were developed.

\section{APPLICABILITY OF THE RISK FUNCTIONS}

All patients entered the study because they had 24 hour electrocardiography. So the models can be interpreted only if 24 hour electrocardiography was performed-that is the model based on history only (model 1 ) is valid in patients in whom 24 hour electrocardiography was indicated but not in those in whom it was not. Because this information is already available it is reasonable to consider model 2 (history, standard 12 lead electrocardiogram, and standard analysis of 24 hour electrocardiogram) as the basic model. We conclude that limited additional information only is gained about the prediction of sudden death if more tests are added to the model. This may have cost-benefit implications for risk stratification after acute myocardial infarction. A high risk for sudden death may be an indicator for treatment, and prevention of sudden death may be attempted in these patients. Furthermore, the risk functions may be used to select high risk patients for intervention studies.

We thank Mr J L H Le Brun and Mrs S van der Does-van der Linden for their help with the retrieval and coding of the patients' records, and the cardiology staff of the University Hospital Rotterdam-Dijkzigt, the Bergwegziekenhuis, the Sint Franciscus Gasthuis, and the Zuiderziekenhuis, Rotterdam for their cooperation.

Supported by grants 83.075 and 37.002 from the Netherlands Heart Foundation and a grant from Cardiolab, Rotterdam. This work was presented in part at the $12^{\mathrm{t}}$ Congress of the European Society of Cardiology, Stockholm, September 1990 and published in abstract form.

1 Kannel W, Thomas HE Sudden coronary death: the Framingham study. Ann NY Acad Sci1982;382:3-21. Kraming WB Schatzkin A. Sudden death: lessons from subsets in population studies. $f \mathrm{Am}$ Coll Cardiol subsets in popul

3 Algra A, Tijssen JGP, Roelandt JRTC, Pool J, Lubsen J. QTc prolongation measured by standard 12 lead electrocardiography is an independent risk factor for sudden death due to cardiac arrest. Circulation 1991:83 1888-94.

4 Algra A, Tijssen JGP, Roelandt JRTC, Pool J, Lubsen J. Heart rate variability from 24-hour electrocardiography and the two-year risk for sudden death. Circulation 1993:88:180-5. 
5 Algra A, Tijssen JGP, Roelandt JRTC, Pool J, Lubsen J. QT interval variables from 24 hour electrocardiography and the two year risk of sudden death. Br Heart $\mathcal{F} 1993$; 70:43-8.

6 Harrell FE. The LOGIST procedure. In: Hastings RP, ed. SUGI supplemental library user's guide. Version 5 edition. Cary, NC: SAS Institute Inc, 1986:269-93.

7 Kleinbaum DG, Kupper LL, Morgenstern H. Epidemiologic research, principles and quantitative Epidemiologic research, principles and quantitative
methods. Belmont, California: Lifetime learning publimethods. Belmont, Calit
cations, 1982;443,71.

8 Weinstein MC, Fineberg HV. Clinical decision analysis. Philadelphia: WB Saunders, 1980;114-20.

9 Voogd PJ, Rijsterborgh H, Lubsen J, Arntzenius AC, Monsjou LK, Godijn EH. Reference ranges of echocardiographic measurements in the Dutch population. Eur Heart J 1984;5:762-70.

10 Prineas RJ, Crow RS, Blackburn H. The Minnesota Code. Manual of electrocardiographic findings. Standards and procedures for measurement and classification. Boston: John Wright, 1982.

11 Rautaharju PM, Warren JW, Jain U, Wolf HK, Nielsen CL. Cardiac infarction injury score: an electrocardiographic coding scheme for ischemic heart disease. graphic coding scheme for
Circulation $1981 ; 64: 249-56$.

12 Kannel WB, Thom TJ. Incidence, prevalence, and mortality of cardiovascular diseases. In: Hurst JW, ed. The heart, arteries and veins. New York: McGraw-Hill, 1986;557-65.

13 Hollifield JW. Electrolyte disarray and cardiovascular disease. Am $\mathcal{F}$ Cardiol 1989;63:21B-6.

14 Velema JP. Prediction of cardiac death-An epidemiological study on the prognostic significance of 24-hour ECG-recordings. Erasmus University Rotterdam, 1982. Doctorate thesis.

15 Schneider JF, Thomas E, Kreger BE, McNamara PM, Kannel WB. Newly acquired left bundle-branch block: the Framingham study. Ann Intern Med 1979;90: the Framin 10 .

16 Rabkin SW, Mathewson FAL, Tate RB. Natural history of left bundle-branch block. Br Heart $\mathcal{F}$ 1980;43:164-9.

17 Lampert S, Lown B, Graboys TB, Podrid PJ, Blatt CM. Determinants of survival in patients with malignant ventricular arrhythmia associted with coronary artery disease. Am F Cardiol 1988;61:791-7.

18 Bigger JT, Fleiss JL, Kleiger R, Miller JP, Rolnitzky LM, and the Multicenter Post-Infarction Research Group. The relationships among ventricular arrhythmias, left ventricular dysfunction, and mortality in the 2 years after myocardial infarction. Circulation 1984;69:250-8.

19 Krone RJ, Gillespie JA, Weld FM, Miller JP, Moss AJ, and the Multicenter Post-Infarction Research Group. Low-level exercise testing after myocardial infarction: Low-level exercise testing after myocardial infarction:
usefulness in enhancing clinical risk stratification. usefulness in enhancin
Circulation 1985;71:80-9.

20 Ruberman W, Weinblatt E, Goldberg JD, Frank CW, Shapiro S. Ventricular premature beats and mortality after myocardial infarction. $N$ Engl $f$ Med 1977;297: $750-7$.

21 Moss AJ, Davis HT, DeCamilla J, Bayer LW. Ventricular ectopic beats and their relation to sudden and nonsudden cardiac death after myocardial infarction. Circulation 1979;60:998-1003.

22 Mukharji J, Rude RE, Poole K, et al. Risk factors for sudden death after acute myocardial infarction: two-year follow-up. Am f Cardiol 1984;54:31-6.

23 Velema JP, Lubsen J, Pool J, Hugenholtz PG. Can cardiac death be predicted from an ambulatory 24-hour ECG? death be predicted from an

24 Myerburg RJ, Kessler KM, Bassett AL, Castellanos A. A biological approach to sudden cardiac death: structure, function and cause. Am $\mathcal{F}$ Cardiol 1989;63:1512-6.

25 Algra A. Electrocardiographic risk factors for sudden death. A study with 245 cases of sudden death during a two-year follow-up after 24-hour electrocardiography in 6693 patients. Erasmus University Rotterdam, 1990. Doctorate thesis.

26 Ripley KL, Okkerse RJ, Engelse WAH, Vinke RVH, Zeelenberg C. Implementation of ARGUS/2H at the Thoraxcentrum. Proceedings of the seventh meeting of Thoraxcentrum. Proceedings of the seventh meeting of
Computers in Cardiology. Long Beach, California: Computers in Cardi

27 Algra A, Le Brun H, Zeelenberg C. An algorithm for computer measurement of QT intervals in the 24 hour ECG. Proceedings of the thirteenth meeting of Computers in Cardiology. Long Beach, California: IEEE, 1986:117-9.

28 Bazett HC. An analysis of the time-relations of electrocardiograms. Heart fournal 1920;7:353-70.

29 Walker SH, Duncan DB. Estimation of the probability of an event as a function of several independent variables. Biometrika 1967;54:167-79.

30 Maisels MJ, Gifford K, Antle CE, Leib GR. Jaundice in the healthy newborn infant: a new approach to an old problem. Pediatrics 1988;81:505-11.

\section{Appendix A}

COMPUTER-AIDED ANALYSIS OF THE 24 HOUR ECG

A stream of all $R R$ intervals was obtained during computer-aided analysis of the 24 hour electrocardiograms. ${ }^{26}$ Selected indices of $R R$ interval duration and variation are shown in the upper part of table 4 . For the calculation of the $R R$ indices only $R R$ intervals between QRS complexes of supraventricular origin were used. Intervals with a duration of $<80 \%$ or $>120 \%$ of that of the running RR average were excluded to eliminate intervals related to premature supraventricular complexes and ventricular arrests. Also excluded were the intervals following a short interval (presumably related to a premature supraventricular complex) because these intervals tend to be prolonged as a partially compensatory pause.

QT intervals were measured during automated computer-aided analysis and were reviewed and corrected manually. ${ }^{27}$ The lead in which QT interval duration showed the strongest correlation with RR interval duration was selected for QT editing, because the quality of the QT measurements in this lead was considered to be best. Three half hour episodes were selected to limit editing time. From the 48 half hour episodes we selected the episode with maximum short-term RR variability, the episode with maximum QT interval variability (the standard deviation of all QT intervals in a half hour episode), and the episode with the shortest TQ intervals (average of all TQ intervals in a half hour episode). QT intervals not selected for review were adjusted with the mean difference of QT measurements before and after review in the three half hour episodes in that 24 hour electrocardiogram.

All QT data analyses were performed with the use of heart rate corrected QT (QTc) intervals. ${ }^{28}$ The definitions of the parameters of QTc interval duration and variation are shown in the lower part of table 4. QTc was calculated only if the current and previous QRS complex were of supraventricular origin. A running $R R$ average with $1 / 8$ update was used in the computation.

\section{Appendix B}

LOGISTIC REGRESSION FUNCTION

With the logistic regression function the conditional probability of sudden death given a set of variables $x_{1}, x_{2}, \ldots, x_{i}$ measured at the time of 24 hour electrocardiography is estimated. The function has the form $P=[1+$ $\left.\exp -\left(a_{0}+b_{1} x_{1}+b_{2} x_{2}+\ldots+b_{j} x_{j}\right)\right]^{-1}$ where $P$ is the probability for sudden death when $x_{1}$, $x_{2}, \ldots, x_{j}$ and $a_{0}, b_{1}, b_{2}, \ldots, b_{j}$ are coefficients to be estimated from the data. Estimation is by the maximum likelihood method of WalkerDuncan. ${ }^{29}$ Constant $a_{0}$ needs to be adjusted by a correction $a_{1}$ because $a_{0}$ is estimated from the data of a subset of all 6693 study patients. ${ }^{30}$ Correction $a_{1}$ is the natural logarithm of $\left(N_{D} / N_{C}\right) /\left(n_{D} / n_{C}\right)$ in which $N_{D}$ is the number of all cases of sudden death, $N_{C}$ is the number of all non-cases and $n_{D}$ and $n_{C}$ are respectively the number cases and noncases in the model. The absolute risk for an individual patient is obtained by calculation of the sum of the patients coefficients $b_{1}$, 
$b_{2}, \ldots, b_{j}$, the adjusted constant $a$ and transforming it into the absolute risk as follows: $P=1 /\left[1+\exp -\left(a+b_{1} x_{1}+b_{2} x_{2}+\ldots+b_{j} x_{i}\right)\right]$.

Besides absolute risk estimates in each model relative risk estimates or odds ratios are obtained directly from the regression coefficients $b_{1}, b_{2} \ldots, b_{j}$. If indicator variables are used, which have a value of 1 if the prop- erty considered is present and 0 if it is not, coefficient $b_{n}$ of variable $x_{n}$ can be interpreted as the logarithm of the relative risk for sudden death of the presence versus the absence of variable $x_{n}$. This relative risk is independent of the other variables retained in the model. It should be noted that this interpretation is conditional upon the data coding strategy. 